CAHIERS DE

NARRATOLOGIE

\section{Cahiers de Narratologie}

Analyse et théorie narratives

$26 \mid 2014$

Nouvelles frontières du récit. Au-delà de l'opposition entre factuel et fictionnel

\title{
Fictions du réel : le journalisme narratif
}

Nicolas Pélissier et Alexandre Eyriès

\section{OpenEdition}

Journals

Electronic version

URL: http://journals.openedition.org/narratologie/6852

DOI: 10.4000/narratologie.6852

ISSN: 1765-307X

Publisher

LIRCES

\section{Electronic reference}

Nicolas Pélissier et Alexandre Eyriès, "Fictions du réel : le journalisme narratif », Cahiers de

Narratologie [Online], 26 | 2014, Online since 14 October 2014, connection on 15 November 2019. URL : http://journals.openedition.org/narratologie/6852 ; DOI : 10.4000/narratologie.6852

This text was automatically generated on 15 November 2019.

Article L.111-1 du Code de la propriété intellectuelle. 


\title{
Fictions du réel : le journalisme narratif
}

\author{
Nicolas Pélissier et Alexandre Eyriès
}

1 Depuis son apparition dans nos sociétés occidentales et son installation tumultueuse dans nos univers de référence et nos pratiques de consommation de biens culturels, le journalisme n'a eu de cesse d'étendre chaque jour un peu plus son champ de compétences, de repousser les frontières de son domaine d'expertise... au risque d'esquisser des rapprochements avec des domaines et des formes d'expressions connexes (la littérature au premier chef) et de s'aventurer sur des terrains sinon vierges du moins mal balisés.

2 C'est ainsi que, loin de se limiter à une simple retranscription des faits et des évènements authentifiés, le journalisme s'est progressivement assigné une mission supplémentaire : celle de raconter des histoires pour adoucir la description sèchement objective du réel. Pour en rendre compte avec transparence et fidélité, les journalistes ont néanmoins besoin de filtres qui viennent s'intercaler entre le réel et la représentation que l'on en donne. Ces filtres constituent « un contrepoint à la nudité et la crudité de la réalité ${ }^{1}$. Au même titre que les journalistes, les juristes savent parfaitement que les faits constituent une épreuve qui implique une préparation, un important effort d'habillage et de mise en scène. C'est cette nécessité de parer le réel des oripeaux de la fiction pour mieux le restituer qui constitue le vecteur à partir duquel s'est élaborée une forme spécifique de journalisme, le journalisme narratif, que nous proposons de définir comme une pratique d'écriture journalistique qui utilise consciemment et à dessein les ressources de la fiction pour analyser et interpréter des faits et les retransmettre dans un second temps à un public. Dans la lignée des travaux de Jean-Michel Adam sur le prototypage des différents types textuels et sur leur séquentialité ${ }^{2}$, Marc Lits amène à son tour un complément de définition en écrivant que "certains énoncés extraits du discours journalistique pourraient prendre la forme d'un type narratif lorsque les six critères suivants sont réunis: une succession d'événements, une unité thématique, des prédicats transformés (...), un procès (c'est-à-dire une action qui forme un tout, comprenant un début, un noud et un dénouement), une causalité narrative qui excède 
l'enchaînement chronologique, une évaluation finale configurante ${ }^{3}$ ». Cette première phase définitionnelle appelle aussitôt un éclairage, car tout article de journalisme narratif induit un changement de paradigme dans les pratiques culturelles et dans les habitudes de consommation du lectorat. Celui-ci se retrouve en effet confronté à des modalités nouvelles de réception auxquelles il n'avait jamais, jusqu'alors, été confronté. Avec l'avènement du journalisme narratif, "le travail du lecteur ne se limite pas à suivre la succession des événements, à juxtaposer des séquences (...), mais à organiser les informations qu'il reçoit par un travail cognitif pour dégager un sens global ${ }^{4} »$. Le journalisme narratif est un modèle journalistique particulier, produisant «des récits hybrides aux frontières $d u$ journalisme et de la littérature. Il se manifeste aujourd'hui dans la sphère journalistique francophone, où il s'inscrit en filiation avec le grand reportage tout en s'affichant comme inspiré du narrative journalism américain ${ }^{5} »$. Par une contextualisation bienvenue, Marie Vanoost rappelle l'importance du phénomène du journalisme narratif dans l'univers anglosaxon et donne à lire une définition américaine de ce phénomène, produite au cœur de l'Université Harvard : "the genre that takes the techniques of fiction and applies them to nonfiction. The narrative form requires deep and sophisticated reporting, an appreciation for storytelling, a departure from the structural conventions of daily news, and an imaginative use of language ${ }^{6 "}$.

3 Cette définition a le grand mérite de mettre l'accent sur l'utilisation de certaines techniques empruntées au récit littéraire dans le cadre des pratiques d'écriture journalistiques. Elle souligne aussi que l'exercice du journalisme narratif nécessite un long travail d'enquête préalable pour parvenir à la forme rendant le plus précisément compte de la réalité observée. Ce recours s'explique, du côté des journalistes, par un intérêt accru pour la pratique de la narration, par un goût avéré pour «l'art de raconter des histoires ${ }^{7}$ » que l'on désigne également sous l'appellation de storytelling, pratique à laquelle Christian Salmon a consacré, en 2007, un ouvrage qui a connu un grand succès éditorial et critique. Se lancer dans une telle forme de journalisme exige de se décentrer totalement des conventions habituelles de traitement et d'exploitation de l'information brute.

4 Le journalisme narratif repose, enfin, sur un travail approfondi sur le langage qui mobilise les ressources de l'imaginaire et de la culture de leur auteur. "Malgré les nuances, des constantes s'imposent donc : pour traiter d'un sujet réel, les auteurs mettent en place des démarches de reportage, puis utilisent des techniques littéraires lors de l'écriture, donnant ainsi à leur texte une portée qui tend à dépasser celle du journalisme « conventionnel ${ }^{8}$ ».

5 A la lumière de ce constat, nous proposons à notre tour d'inscrire le journalisme narratif dans une zone frontière, dans un "entre-deux narratif ", dans lequel l'impératif réaliste de l'éthique journalistique n'entrave pas la célébration du style et la recherche $\mathrm{du}$ suspense via des procédés de mise en tension narrative ${ }^{9}$. De fait, son mode d'écriture est beaucoup moins contraint par les formats les plus usuels (genres, nombre de signes, balises de lecture...) du journalisme plus factuel. Le narrateur y manifeste plus activement sa présence et son implication. Enfin, la quête du style et du suspense est facilitée par une prise de distance vis-à-vis du modèle canonique et de ses leitmotivs : usage des " $5 \mathrm{~W}$ », de séquences chronologiques (temps linéaire), recherche de motsclés, de concision du propos et, surtout, d'une neutralité posée comme impératif catégorique. Le journalisme narratif a progressivement conquis sa liberté par rapport aux normes... mais pas au point de s'en affranchir. Même s'il utilise à son avantage les ressources de l'écriture de fiction (romanesque notamment), il demeure avant tout un 
récit du réel (non fiction writing), ce qui détermine en large partie ses formes de narration et ses formats scripturaux spécifiques. Le récit journalistique entretient avec le monde réel dont il se fait l'écho un lien ambivalent puisqu'il en constitue aussi une retranscription, une recomposition, une réinvention.

$6 \mathrm{Au}$ terme de cette première entreprise de définition des enjeux, des formes et des pratiques du journalisme narratif, nous proposons dans cet article une réflexion croisée sur le journalisme narratif et le storytelling (et leur relation ambigüe au factuel et au fictionnel), puis des analyses ciblées d'articles de journalisme narratif issus de la revue XXI pour en souligner les spécificités scripturales et les implications éthiques.

\section{Journalisme narratif et storytelling : regards croisés}

7 Le journalisme narratif privilégie les stories sur les news et mobilise les ressources des récits avec un souci constant d'accuracy (appelant une vérification minutieuse de l'exactitude des faits rapportés). Il emprunte des chemins différents et complémentaires que l'on retrouve dans certaines formes de "grand reportage ». Il permet ainsi de «situer le travail du journaliste sur des terrains désertés ou oubliés par l'information à flux tendu : l'immersion, le témoignage des acteurs, l'observation y jouent alors un rôle essentie ${ }^{10}$ ». Le modèle journalistique narratif privilégie les détails concrets et le vécu en temps réel de l'événement en train de se dérouler. Il met en scène des personnages qui dialoguent entre eux au fil d'un véritable scénario du réel comportant un début, une fin et des épisodes de mise en tension du lecteur ${ }^{11}$. Le narrateur se fond dans le décor, il devient acteur du milieu observé, ce qui lui permet d'affûter son regard et sa perception de l'environnement. Au final, il contribue à ré-enchanter un monde que le journalisme factuel enferme au quotidien dans des contenants toujours plus petits et étanches.

8 Un tel retour du récit s'est opéré sous l'impulsion du New Journalism ${ }^{12}$, qui puise son inspiration dans l'écriture poétique, musicale, littéraire et cinématographique. Ses auteurs (Mailer, Thompson) flirtent volontiers avec le lyrisme. Ils privilégient le style, la subjectivité de la mise en scène, la déconstruction du temps, l'interpellation du lecteur et la production d'images choc, sous l'emprise de substances souvent illicites (comme dans Las Vegas parano d'Hunter S. Thompson). La description de la réalité observée trouve des formes d'accomplissement à la fois très déroutantes et abouties. Mais, à force d'utilisations de procédés propres à la fiction, ce "nouveau journalisme » perd parfois tout contact avec le réel, l'empêchant ainsi d'assurer durablement sa légitimité auprès du public et des éditeurs. Les apports du nouveau journalisme ont été des plus féconds et ont donné naissance à un véritable narrative turn dans l'écriture journalistique de ces dernières décennies.

9 A titre d'exemple actuel, on peut mentionner le rôle actif des professionnels de l'information comme des apprentis journalistes sur des plateformes participatives du Web telles que Storify. Le journalisme contribuerait-il donc, à sa manière, au mouvement d'emprise du storytelling décrit par Christian Salmon? L'usage croissant des procédés du récit par les journalistes est-il devenu manipulatoire? Le storytelling journalistique a-t-il sa propre logique et autonomie ou se réduit-il à un relais utile de la «machine à raconter des histoires et à formater les esprits» des organisations marchandes et politiques? 
10 En nous référant aux travaux académiques traitant de ces questions, il apparait que les critiques de la narration journalistique ne manquent pas, notamment dans la recherche française en sciences de l'information et de la communication. Cependant, l'usage du récit par les journalistes n'est pas en soi condamnable et il existe bien un journalisme narratif pouvant constituer un contre-pouvoir à celui des narrations hégémoniques de ces grandes organisations visées par Salmon. Dans les années 1990 (à l'occasion de la guerre du Golfe, notamment), le discours des journalistes est de plus en plus perçu comme un élément d'une stratégie visant à leurrer le public en utilisant massivement la communication : en attirant l'attention du public sur des éléments de décor, il détourne son attention des aspects les plus dramatiques de la guerre, notamment la destruction et la mort ${ }^{13}$. Au prisme déformant de la télévision, l'idée d'une " guerre propre » n'est plus seulement un discours d'état-major. Elle devient la réalité pour un téléspectateur dont l'esprit critique a été anesthésié à grand renfort d'images. Le journalisme narratif est une appellation d'une grande plasticité qui "peut être utilisée, plus facilement que d'autres, pour désigner autant des textes écrits que d'autres types de productions journalistiques, de la BD-reportage au webdocumentaire. Dans le contexte médiatique et journalistique actuel de concurrence généralisée et de nouvelles hybridations, notamment liées aux nouvelles technologies ${ }^{14} "$, la souplesse du concept de journalisme narratif recouvre une multiplicité d'expériences et de pratiques qui ressortissent toutes, à différents égards, à un usage de la narration utile (ou storytelling) qui est mise au service du traitement de l'information brute qui est délivrée par le fil AFP ou les dépêches d'agence dans les salles de rédaction. Il n'est donc pas du tout innocent, en fin de compte, que le journalisme narratif soit considéré comme potentiellement ou effectivement manipulatoire. Ses narrations sont perçues comme des amplificateurs équivoques du storytelling, des instruments de domination symbolique et de valorisation marchande. A force de raconter et de se raconter des histoires, les journalistes se déconnectent de la réalité. Ils vont croire eux-mêmes et faire croire aux « histoires vraies " qu'ils ont écrites pour le public. La médiatisation récente et hasardeuse de certaines affaires judiciaires (celle d'Outreau, par exemple) en offre une tragique illustration.

11 Nous souhaitons à présent considérer le journalisme narratif sous un angle plus critique et interroger, notamment, la tentation de la fiction (et l'inclination pour le storytelling) à laquelle cèdent parfois des journalistes aguerris. A la différence des partisans du New Journalism, volontiers attirés par la fiction, les représentants du New New Journalism consacrent en effet un retour du réel dans la narration journalistique. Il s'agit moins, pour eux, de rechercher le style que de coller au plus près du vécu de trajectoires humaines individuelles et collectives. Ce "néo-réalisme journalistique » utilise de tels procédés pour mieux décrire l'actualité à l'abri des normes les plus contraignantes de l'industrie des médias classiques, mais aussi pour mieux « porter la plume dans la plaie » et révéler des injustices sociales souvent occultées par ces médias. Dans cette optique, ils s'investissent sur un temps long, à la manière des anthropologues, dans l'exploration en profondeur d'une situation ou d'une communauté souvent marginalisée. C'est le cas d'Eric Schlosser, qui s'intéresse aux consommateurs des fast-foods ou d'Adrian Nicole Leblanc, qui s'immerge dans l'univers bigarré des Portoricains du Bronx. En France, Florence Aubenas se plonge dans le milieu des travailleurs précaires du Quai de Ouistreham, et Arthur Frayer dans celui, peu engageant, des gardiens de prison. 
12 Le concept d'«hypernarrativité » forgé par Marc Lits s'applique particulièrement bien aux nouvelles formes de co-construction des récits journalistiques par les « lect-acteurs " (ou prod-users) de l'information en ligne au moyen de dispositifs socio-techniques participatifs (Huffington Post) : "la dimension narrative est, [...] grâce aux éléments de circularité et d'altérité présents à la fois chez Ricour et chez Bakhtine, de plus en plus dans l'instance de réception qui fonde, elle, son récit propre dans une polyphonie énonciative ${ }^{15} »$. Ces dispositifs permettent des pratiques de crowdsourcing et favorisent l'invention d'un storytelling journalistique beaucoup plus favorable aux lecteurs. Grâce à ce storytelling, les lecteurs ont la possibilité de se réapproprier les sources, les modes d'écriture, mais aussi les pratiques de hiérarchisation de l'information autrefois réservées aux professionnels des rédactions. Certains outils de « curation » tels que Storify permettent en outre aux journalistes professionnels, en collaboration avec leurs confrères, mais aussi avec des internautes ordinaires, d'expérimenter de nouvelles techniques narratives hypertextuelles au service de la couverture de l'actualité ${ }^{16}$. On retrouve ici une hypothèse formulée par Christian Salmon lorsqu'il évoque le surgissement de contre-récits susceptibles de concurrencer le « rouleau compresseur du réel » produit par le storytelling dominant: "cette lutte a déjà commencé, elle se fraye un chemin dans le tumulte d'Internet et le désordre des stories, elle s'éveille à des pratiques nouvelles et minoritaires, échappant largement au regard des médias dominants ${ }^{17} »$. Cette assertion de Salmon appelle aussitôt une précision: les pratiques mentionnées sont aussi portées, aux Etats-Unis comme en Europe, par des représentants de ces médias classiques, évoluant aux marges de leurs organisations. Et l'on ne peut que regretter que Salmon passe sous silence le rôle du journalisme et des journalistes dans la reconquête narrative qu'il se prend à prophétiser.

13 Devons-nous pour autant en conclure que le journalisme narratif est une panacée apte à sortir le journalisme de la crise structurelle qu'il traverse? Rien ne nous y autorise. S'il présente de nombreux atouts, le journalisme narratif ne peut cependant pas, à lui seul, compenser les dysfonctionnements de la machinerie médiatique contemporaine. Par ailleurs, il faut se garder d'un "tout narratif " qui pourrait détourner les journalistes de leurs missions déontologiques les plus fondamentales. Nous pensons donc que le journalisme narratif peut constituer un contrepoint aux dérives du storytelling... mais sous certaines réserves.

14 La première réserve porte sur le nécessaire renforcement des compétences narratives des journalistes, qui relèvent davantage de l'acquis que de l'inné et qui pourraient faire l'objet d'un approfondissement au cours de la formation professionnelle. Or, la place de l'étude et de la pratique du récit est rare et disparate dans les écoles de journalisme. C'est la raison pour laquelle nous suggérons un rapprochement progressif des formations aux humanités (lettres, arts et sciences humaines) de celles qui préparent aux métiers du journalisme, sachant que ces deux types de formations ont été fortement découplés depuis un quart de siècle. Les étudiants en journalisme évoluent dans un monde académique à part, le plus souvent déconnecté de celui des arts et lettres, là où sont enseignées, notamment, les techniques du récit de fiction. L'actuelle convergence "médiaculturelle ${ }^{18}$ " nous amène au contraire à penser ensemble ces différentes composantes du savoir.

15 La seconde réserve concerne tant les établissements de formation que les entreprises de presse qui sont rompues à la fabrique en série de "petits soldats du journalisme " aptes à réécrire dans l'urgence les dépêches des agences. Centres de formation et 
organes de presse gagneraient sans nul doute à accorder plus d'importance aux pratiques immersives sur le terrain, aux méthodes d'investigation, dans le cadre de formats et de temps rallongés. A l'heure de l'emballement généralisé de la production de nouvelles, une certaine forme de ralentissement serait la bienvenue. Elle seule peut garantir la phase 3 de « refiguration » du récit valorisée par Ricœur, celle de la réappropriation par le lecteur.

Ce qui amène à évoquer une troisième réserve : une meilleure prise en compte par les journalistes des attentes et compétences du public. Sans cette prise en compte, l'écriture journalistique risque de tourner à vide. Les blogs et les sites participatifs offrent d'innombrables matrices narratives aux lecteurs, leur permettant de contribuer au récit journalistique et de le recomposer, de se l'approprier. Mais ce pari n'est pas gagné d'avance, tant s'en faut. Nous plaidons donc, en sens inverse, pour une généralisation des enseignements des techniques d'écriture et d'introduction aux genres journalistiques dans des formations humanistes plus généralistes. Il s'agit d'améliorer les qualités rédactionnelles des étudiants, mais, surtout, de préparer les citoyens à décoder les narrations journalistiques et à se les réapproprier au moyen des outils du web collaboratif. C'est, d'ailleurs, l'objectif de l'hypernarratologie théorisée par Marc Lits et l'Observatoire du Récit Médiatique. Cette approche «croisée avec la prise en compte des avancées technologiques, des supports nouveaux, des évolutions des usages et des publics, [...] permettra de saisir l'homme socialisé comme un animal narrativisé, traversé par des récits construits selon des formes radicalement nouvelles et ouvertes ${ }^{19}$ ». Enfin, nous estimons qu'un journalisme narratif contrepoids au storytelling dominant ne peut s'épanouir sans un souci de vigilance réaliste de la part de ses praticiens et promoteurs. Trouver un équilibre entre cet impératif et celui de l'engagement civique (responsabilité sociale du journalisme) ou celui de l'inévitable mise en spectacle de l'information, n'est pas chose aisée. S'il est bien une construction sociale, le journalisme narratif n'est pas une fiction parmi tant d'autres, il s'en sert éventuellement (et même assez fréquemment, au même titre que d'autres dispositifs sociotechniques et que bien d'autres outils) pour accomplir sa tâche de relation et de restitution des faits.

$17 \mathrm{Au}$ terme de cette partie consacrée aux rapports complexes et contrariés du journalisme narratif - auquel on a pu reprocher de trahir le réel et les faits à force de s'inspirer de la fiction - et du storytelling - synonyme, pour beaucoup, de manipulation - nous proposerons ensuite une réflexion sur les modalités d'écriture spécifiques au journalisme narratif (et aux matrices narratives qu'il mobilise) à partir d'exemples issus de la revue XXI (constituant actuellement en France la publication la plus représentative du champ du journalisme narratif).

\section{Le journalisme narratif : le creuset d'un roman du réel ?}

18 Dans le cadre de l'écriture journalistique, l'usage du récit et le recours aux techniques empruntées à la fiction littéraire ne vont pas de soi, ce ne sont pas des démarches naturelles pour un journaliste - fût-il expérimenté -, elles impliquent une véritable acculturation. Le journalisme narratif constitue une expérience de décentrement par rapport aux pratiques habituelles. Il s'inscrit en rupture avec les règles d'écriture classiques (phrases courtes, lisibilité immédiate, pyramide inversée, 5W) et avec les normes enseignées dans les écoles de journalisme et les manuels pratiques à 
destination de ceux qui veulent embrasser la profession. La revue XXI, qui a été parmi les première revue-livres (ou mooks) lancées en Europe, accueille en France "tous les talents $d u$ reportage $e^{20} »$. Le reportage constitue, par essence, l'un des lieux privilégiés de la prise de distance avec les règles scripturales classiques. C'est d'ailleurs une constante et une caractéristique héritées de la longue tradition du grand reportage de facture « littéraire » qu'ont pu incarner, à une certaine époque en France, des journalistes et écrivains aussi célèbres et différents qu'Albert Londres (avec des ouvrages tels que $\mathrm{Au}$ bagne, 1923; Chez les fous, 1925), Gaston Leroux ( auteur du livre intitulé L'agonie de la Russie blanche, 1928) ou encore Joseph Kessel (En Syrie, 1927; Avec les Alcooliques Anonymes, 1960 ; Terre d'amour et de feu. Israël, 196521).

Afin de mettre en évidence les composantes de ce modèle journalistique et les raisons manifestes de son récent retour en grâce, nous analyserons un article paru en juillet 2012 dans la revue XXI ( L La confession d'un prêtre tueur ») qui porte en germe à la fois toutes les caractéristiques du genre et les ferments d'un renouveau des pratiques d'écriture narrative que de nombreux professionnels de l'information appellent de leurs vœux.

Dans cet article, le journaliste et réalisateur Thomas Dandois retrace sa première rencontre en Colombie avec Jorge Léon Delgado, ancien tueur à gages devenu d'abord trafiquant de drogue puis pasteur évangéliste. Dès le titre de l'article, le champ lexical de la religion est convoqué par le biais d'une figure de style dérivative puisque le " prêtre » est un ancien " tueur " à gages professionnel et que sa "confession » n'est pas faite devant un ministre du culte dans le confessionnal d'une église mais devant les hommes et plus particulièrement devant des magistrats et des jurés au sein d'une cour de justice. Le journaliste manifeste ainsi sa capacité à se servir avec brio de la dimension polysémique du langage.

21 En tant que pasteur, l'ancien tueur professionnel «arpentait tous les jours les rues de Siloé, un quartier pauvre et violent de Cali, la troisième ville de Colombie. Là où la police n'entrait qu'armée et en gilets pare-balles, il avançait avec sa petite sacoche et son sourire en bandoulière pour tendre des petits papiers surs lesquels était écrit: "Dieu t'aime. Même au fond des ténèbres, il t'appelle". [...] Nul n'osait se mettre en travers du chemin du professionnel repenti2 ${ }^{22}$ ». Cette entame d'article est digne des meilleurs romans policiers, d'emblée on retrouve certains invariants caractéristiques de ce genre littéraire (courage physique, charisme, dangerosité du personnage, capacité à se défendre, etc.), une atmosphère reconnaissable entre toutes.

22 La construction même du récit révèle l'habileté scripturale du journaliste qui recourt volontiers à des analepses (en d'autres termes des retours en arrière, l'équivalent des flashbacks au cinéma) - « les bras posés sur la table, Jorge plonge en lui-même pour remonter le fil, repartir dans ce passé qu'il a voulu racheter ${ }^{23}$ »- mais aussi à des prolepses (figure d'anticipation, qui est ce que l'on retrouve dans les fictions audiovisuelles sous la forme de flashforwards) : «quatre ans plus tard - il vient d'avoir 14 ans -, il retrouve son agresseur et le coince dans une rue déserte. "Des jeunes du quartier m'avaient prêté un pistolet P38. J'ai descendu le gars, ça m'a libéré". Sans état d'âme, il retourne à son quotidien de petit vendeur de cacahuètes $^{24}$ »- pour maintenir son lecteur en haleine, dans un état de concentration maximal. Les aventures de Jorge se lisent comme un roman et sont d'autant plus passionnantes qu'elles sont rigoureusement authentiques.

23 L'article de Thomas Dandois prend la forme d'une narration rythmée et maitrisée qui retrace la vie à feu et à sang de Jorge Léon Delgado qui sème la mort autour de lui 
durant dix ans et dépense tout son argent avec la drogue, les femmes et les fêtes dans les bars des quartiers mal famés. Avec un sens aigu du rythme et de la narration, le journaliste décrit un personnage implacable et sans pitié devant l'arme duquel «des hommes tombent à genoux et appellent leur mère, de jeunes adolescents s'oublient de peur. Personne ne trouve grâce. "Mon cœur était de pierre. Je ne voyais plus d'hommes en face de moi, juste des contrats $25 " »$. En recourant aux techniques de la fiction romanesque, le journaliste parvient à recréer l'itinéraire criminel de Jorge, à pallier les carences de sa mémoire, à combler les vides du récit. Là intervient le talent incomparable du narrateur qui se substitue au journaliste pour imaginer le cadre et le train de vie de son protagoniste pourtant bien réel : «la famille vit dans le voisinage des riches entrepreneurs et des stars des telenovelas, les séries télé. La maison, carrelée en imitation marbre, compte deux étages. Jorge mène grand train: motos, grosses voitures, montres en or, femmes de ménage ${ }^{26}$. Comme dans un roman - mais alors, bien sûr, un roman du réel - , le tueur trafiquant de drogue devient pasteur à la grande satisfaction de ses paroissiens et sillonne le quartier le plus dangereux de la ville. Son passé de tueur s'estompe jusqu'au jour où une balle lui transperce la nuque sans le tuer miraculeusement. Il est tout de même rattrapé par la justice, condamné à vingt ans de prison et libéré au bout de sept. Renonçant à être pasteur, il devient marchand ambulant et fait plusieurs fois face à la violence sans replonger : "Jorge n'a pas eu besoin de tuer. Pas cette fois. "Je veux vivre en paix", murmure-t$i^{27} »$.

\section{Conclusion}

$\mathrm{Au}$ terme de cet article, nous souhaitons montrer que le journalisme narratif fait dialoguer la voix individuelle et la voix narrative et se construit entre la nécessaire précision factuelle et l'exigence scripturale du récit. Il constitue un mode particulier de remise en cause de la traditionnelle opposition entre factuel et fictionnel, et il nous faut ici préciser qu'en aucune manière, le choix de la forme narrative n'exempte le journaliste d'une phase classique de recueil de données. Le reportage solidement étayé, la recherche documentaire dans des bases de données, l'investigation minutieuse menée sur une temporalité étendue, l'immersion dans un milieu (fût-il interlope comme celui du crime organisé, des réseaux de prostitution ou cynique comme celui de l'industrie agro-alimentaire) sur une longue période sont des préalables nécessaires à toute narration journalistique. Le récit, dans le journalisme narratif, constitue une mise en ordre et en mots fondamentale des fragments du réel qui ont été recueillis sur le terrain.

Face au storytelling hégémonique (qui fonctionne comme une machine à fabriquer des histoires en série), le journalisme narratif peut constituer une alternative pertinente, à une seule condition : celle que ses praticiens et ses farouches partisans manifestent en toutes circonstances un souci de vigilance réaliste. En effet, s'il est bien une construction sociale, le journalisme n'est pas pour autant une simple fiction. Il se sert du récit pour accomplir sa restitution des faits. Le modèle de journalisme narratif auquel nous croyons se situe à la croisée des Media Studies et des Cultural Studies.

Dans cette perspective nous nous inscrivons clairement à la suite des travaux de Robert Ezra Park et de Géraldine Mulhmann et considérons le journalisme narratif à la fois comme une forme culturelle à part entière et comme une méthode d'obédience réaliste qui implique un traitement de sang froid du matériau factuel. Car, en définitive, ce que 
produit le journalisme narratif, ce sont bien des fictions, mais des fictions d'un genre particulier : des fictions du réel.

\section{BIBLIOGRAPHY}

Bibliographie

Adam, J-M., (1992), Les textes. Types et prototypes, Paris : Le Seuil.

Baroni R., (2007), La tension narrative : suspense, curiosité, surprise : Paris, Le Seuil.

Boynton S. R., (2005), The New New Journalism : Conversations With America's Best Nonfiction Writers on Their Craft, New York : Vintage Books.

Brin C., Charron J., De Bonville J, (2004), Nature et transformations du journalisme. Théories et recherches empiriques, Québec : Les Presses de l'Université Laval.

Coman M., (2003), Anthropologie des médias, Grenoble : PUG.

Dandois T., (2012), « La confession du prêtre tueur », Revue XXI n¹9, p 32-43.

Charon J-M., et Mercier A., (2004), Armes de communication massive, Paris : CNRS Editions.

Gauthier G., (2004), « La vérité, visée obligée du journalisme », Cahiers du journalisme n ${ }^{\circ}$, printemps 2004, p 164-182.

Grévisse B., (2008), Ecritures journalistiques, Bruxelles : De Boeck / INA.

Hernandez K., (2012), L'évolution du journalisme narratif sur le web : le cas de l'outil Storify, Mémoire de recherche de Master en Sciences de l'Information et de la Communication, Université de Nice Sophia Antipolis.

Leblanc G., (1997), Scénarios du réel, Paris : L’Harmattan.

Lits M., (2008), Du récit au récit médiatique, Bruxelles : De Boeck.

Lallemand A., (2011), Journalisme narratif en pratique, Bruxelles : De Boeck.

Macé E., (2006), Les Imaginaires médiatiques. Une sociologie postcritique des médias, Paris : éditions Amsterdam.

Muhlmann G., (2006), Du journalisme en démocratie, Paris, Payot et Rivages : collection Petite Bibliothèque Payot $n^{\circ} 601$.

Park R.E., (2008), Le journaliste et le sociologue (textes présentés et commentés par Géraldine Mulhmann et Edwy Plenel), Paris, Editions du Seuil : collection Médiathèque.

Pélissier N., (2010), Journalisme, avis de recherches, Bruxelles : Editions Bruylant.

Pélissier N., et Marti M., (dirs.) (2012), Le storytelling, succès des histoires, histoire d'un succès, Paris, L'Harmattan : collection Communication et Civilisation.

Ruellan D., (1993), Le professionnalisme du flou, Grenoble : PUG.

Salmon C., (2007), Storytelling. La machine à fabriquer des histoires et à formater les esprits, Paris : La Découverte. 
Vanoost M., (2013), « Journalisme narratif : proposition de définition, entre narratologie et éthique ", Les Cahiers du Journalisme n²5, Printemps / Eté.

Wolfe T., (1973), The New Journalisme, New York : Harper and Row.

\section{NOTES}

1. N. Pélissier, «Le journalisme narratif: vecteur privilégié du storytelling ou antidote à ses dérives? » dans Marc Marti et Nicolas Pélissier, Le storytelling, succès des histoires, histoire d'un succès, Paris, L'Harmattan, 2012, p.117.

2. J.-M. Adam, Les textes. Types et prototypes, Paris, Le Seuil, 1992.

3. M. Lits, Du récit au récit médiatique, Bruxelles, De Boeck, 2008, p. 73.

4. Ibid., p. 71.

5. M. Vanoost « Journalisme narratif : proposition de définition, entre narratologie et éthique », dans Les Cahiers du Journalisme $n^{\circ} 25$, Printemps/Eté, 2013, p. 145

6. «Narrative journalism», Nieman Foundation for Journalism at Harvard. http:// www.nieman.harvard.edu/NiemanFoundation/ProgramsAndPublications/Narrative

Journalism.aspx, cité par M. Vanoost «Journalisme narratif : proposition de définition, entre narratologie et éthique ", op. cit., p. 141.

7. C. Salmon, Storytelling. La machine à fabriquer des histoires et à formater les esprits, Paris, Editions La Découverte [première édition 2007] collection La Découverte / Poche, 2008, p. 7.

8. M. Vanoost «Journalisme narratif : proposition de définition, entre narratologie et éthique », op. cit., p. 146.

9. R. Baroni, La tension narrative : suspense, curiosité, surprise, Paris, Le Seuil, 2007.

10. M. Marti et N. Pélissier, «Storytelling, une histoire de questions » dans Marc Marti et Nicolas Pélissier (dir.), Le storytelling, succès des histoires, histoire d'un succès, Paris, L'Harmattan : collection Communication et Civilisation, 2012, p. 14.

11. Cf. G. Leblanc, Scénarios du réel, Paris, L'Harmattan, 1997.

12. Cf. T. Wolfe, The New Journalisme, New York, Harper and Row, 1973.

13. Cf. J-M. Charon et A. Mercier., Armes de communication massive, Paris, CNRS Editions, 2004.

14. Ibid., p. 147.

15. M. Lits, «Storytelling: réévaluation d'un succès éditorial» dans Marc Marti et Nicolas Pélissier, Le storytelling, succès des histoires, histoire d'un succès, op.cit., p. 36.

16. Cf. K. Hernandez, L'évolution du journalisme narratif sur le web : le cas de l'outil Storify, Mémoire de recherche de Master en Sciences de l'Information et de la Communication, Université de Nice Sophia Antipolis, 2012.

17. C. Salmon, Storytelling, op. cit., p. 212.

18. E. Macé, Les Imaginaires médiatiques. Une sociologie postcritique des médias, Paris, éditions Amsterdam, 2006.

19. M. Lits, «Storytelling : réévaluation d'un succès éditorial », op.cit., p. 35.

20. http://www.revue21.fr/Le-projet

21. Voir notamment D. Ruellan, Le professionnalisme du flou, Grenoble, PUG, 1993.

22. Thomas Dandois, «La confession du prêtre tueur ", Revue XXI n 19, juillet 2012, p. 33.

23. Ibid., p. 34.

24. Ibid., p. 34 .

25. Ibid., p. 36.

26. Ibid., pp. 36-37.

27. Ibid., p. 41. 


\section{ABSTRACTS}

In this article, we chose initially to start from a paradoxical command (i.e. the need to use techniques of literary fiction to give a better representation of the real world and to account for it) to anchor our study in a particular journalistic practice, knows as "narrative journalism", that we define as a way of writing that deliberately relies on fictional resources to review and comment upon the facts and then, in a second time, make them available to the general public. Then, in a second stage, we develop cross reflection on narrative journalism and storytelling, which have complex and ambiguous relationships with the proven real as well as with fictional invention.

Dans cet article, nous avons choisi dans un premier temps de partir d'une injonction paradoxale (la nécessité de recourir aux techniques de la fiction littéraire pour mieux donner à voir le réel et en rendre compte) pour ancrer notre étude dans une pratique journalistique particulière, le journalisme narratif que nous définissons comme une façon d'écrire qui s'appuie à dessein sur des ressources fictionnelles pour examiner et commenter des faits et les mettre ensuite, dans un second temps, à la disposition du grand public. Nous développerons ensuite, dans un second temps, une réflexion croisée sur le journalisme narratif et le storytelling qui entretiennent des rapports complexes et équivoques aussi bien avec le réel avéré qu'avec l'invention fictionnelle.

\section{INDEX}

Chronological index: XXIe siècle

Mots-clés: journalisme, fiction, narration, réel et fictif, storytelling

\section{AUTHOR}

\section{NICOLAS PÉLISSIER ET ALEXANDRE EYRIÈS}

Laboratoire I3M, Université Nice Sophia Antipolis 